\title{
Some fractional proportional integral inequalities
}

\author{
Gauhar Rahman ${ }^{1}$, Thabet Abdeljawad ${ }^{2 *}$, Aftab Khan ${ }^{1}$ and Kottakkaran Sooppy Nisar ${ }^{3}$
}

"Correspondence:

tabdeljawad@psu.edu.sa

${ }^{2}$ Department of Mathematics and

General Sciences, Prince Sultan

University, Riyadh, Saudi Arabia

Full list of author information is

available at the end of the article

\begin{abstract}
In the last few years, various researchers studied the so-called conformable integrals and derivatives. Based on that notion some authors used modified conformable derivatives (proportional derivatives) to generate nonlocal fractional integrals and derivatives, called fractional proportional integrals and derivatives, which contain exponential functions in their kernels. Our aim in this paper is to establish some new integral inequalities by utilizing the fractional proportional-integral operators. In fact, certain new classes of integral inequalities for a class of $n(n \in \mathbb{N})$ positive continuous and decreasing functions on $[a, b]$ are presented. The inequalities presented in this paper are more general than the existing classical inequalities.
\end{abstract}

MSC: 26A33; 26D10;26D53

Keywords: Fractional integrals; Fractional proportional integrals; Fractional integral inequalities

\section{Introduction}

Fractional calculus is the generalization of derivatives and integrals of arbitrary noninteger order. This field has earned more recognition due to its applications in diverse domains [11, 18, 25]. Recent research has focused on developing a number of fractional integral operators and their applications in multiple disciplines of sciences. In [17], the authors introduced the idea of fractional conformable derivative operators with a shortcoming that the new derivative operator does not tend to the original function when the order $\rho \rightarrow 0$. In [3] the authors studied certain various properties of the fractional conformable derivative operator and raised the problem of how to use conformable derivative operators to generate more general types of fractional derivative operators. Later on, in [8], Anderson and Ulness improved the idea of the fractional conformable derivative by introducing the idea of local derivatives. In $[4,5,7,9,19]$, some researchers defined new fractional derivative operators by using exponential and Mittag-Leffler functions in the kernels. In [16], Jarad et al. introduced the left and right generalized proportional-integral operators which are respectively defined by

$$
\left({ }_{a} \Im^{\beta, \mu} f\right)(x)=\frac{1}{\mu^{\beta} \Gamma(\beta)} \int_{a}^{x} \exp \left[\frac{\mu-1}{\mu}(x-t)\right](x-t)^{\beta-1} f(t) d t, \quad x>a,
$$

(c) The Author(s) 2019. This article is distributed under the terms of the Creative Commons Attribution 4.0 International License (http://creativecommons.org/licenses/by/4.0/), which permits unrestricted use, distribution, and reproduction in any medium, provided you give appropriate credit to the original author(s) and the source, provide a link to the Creative Commons license, and indicate if changes were made. 
and

$$
\left(\mathfrak{I}_{b}^{\beta, \mu} f\right)(x)=\frac{1}{\mu^{\beta} \Gamma(\beta)} \int_{x}^{b} \exp \left[\frac{\mu-1}{\mu}(t-x)\right](t-x)^{\beta-1} f(t) d t, \quad x<b,
$$

where $\mu \in(0,1]$ and $\beta \in \mathbb{C}$ and $\Re(\beta)>0$ and $\Gamma$ is the well-known gamma function.

Remark If we consider $\mu=1$ in (1.1) and (1.2), then we get the left and right RiemannLiouville which are respectively defined as

$$
\left({ }_{a} \Im^{\beta} f\right)(x)=\frac{1}{\Gamma(\beta)} \int_{a}^{x}(x-t)^{\beta-1} f(t) d t, \quad x>a,
$$

and

$$
\left(\Im_{b}^{\beta} f\right)(x)=\frac{1}{\Gamma(\beta)} \int_{x}^{b}(t-x)^{\beta-1} f(t) d t, \quad x<b,
$$

where $\beta \in \mathbb{C}$ and $\Re(\beta)>0$.

Such type of generalizations promotes future research to establish more new ideas to unify the fractional derivative and integral operators and obtain fractional integral inequalities via such generalized fractional derivative and integral operators. Integral inequalities and their applications play a vital role in the theory of differential equations and applied mathematics. A variety of various types of some classical integral inequalities and their generalizations have been established by utilizing the classical fractional integral, fractional derivative operators and their generalizations are found in $[12,13,22,23$, $28,31,32]$. A Gronwall inequality and the Minkowski inequalities via generalized proportional fractional derivative and fractional integral are found in the recent work of Alzabut et al. and Rahman et al. $[6,29]$. The Minkowski's inequality for the AB-fractional integral operator is found in [15].

In [30], Sarikaya and Budak studied the $(k, s)$-Riemann-Liouville fractional integral and applications.

In [24], generalized Hermite-Hadamard type inequalities via fractional integral operators are found. In [2], Agarwal et al. introduced Hermite-Hadamard type inequalities by employing the $k$-fractional integrals operators. In [14], Dahmani introduced certain classes of fractional integral inequalities by utilizing a family of $n$ positive functions. In [1], the authors established fractional integral inequalities for a class of family of $n(n \in \mathbb{N})$ positive continuous and decreasing functions on $[a, b]$ by employing the $(k, s)$-fractional integral operators. Recently the authors [10, 21, 26, 27] introduced various types of inequalities by employing the fractional conformable integrals.

\section{Main results}

In this section, we employ the left generalized proportional fractional integral operator to establish the generalization of some classical inequalities.

Theorem 2.1 Let $g$ be a positive continuous and decreasing function on the interval $[a, b]$. Let $a<x \leq b, \vartheta>0, \sigma \geq \gamma>0$. Then, for generalized proportional fractional integral oper- 
ator (1.1), we have

$$
\frac{{ }_{a} \mathfrak{I}^{\beta, \mu}\left[g^{\sigma}(x)\right]}{{ }_{a} \mathfrak{I}^{\beta, \mu}\left[g^{\gamma}(x)\right]} \geq \frac{a^{\mathfrak{I}^{\beta, \mu}}\left[(x-a)^{\vartheta} g^{\sigma}(x)\right]}{{ }_{a} \mathfrak{I}^{\beta, \mu}\left[(x-a)^{\vartheta} g^{\gamma}(x)\right]},
$$

where $\mu \in(0,1], \beta \in \mathbb{C}$, and $\Re(\beta)>0$.

Proof Since $g$ is a positive continuous and decreasing function on the interval $[a, b]$, we have

$$
\left((\rho-a)^{\vartheta}-(t-a)^{\vartheta}\right)\left(g^{\sigma-\gamma}(t)-g^{\sigma-\gamma}(\rho)\right) \geq 0,
$$

where $a<x \leq b, \vartheta>0, \sigma \geq \gamma>0$, and $t, \rho \in[a, x]$.

By (2.2), we have

$$
(\rho-a)^{\vartheta} g^{\sigma-\gamma}(t)+(t-a)^{\vartheta} g^{\sigma-\gamma}(\rho)-(\rho-a)^{\vartheta} g^{\sigma-\gamma}(\rho)-(t-a)^{\vartheta} g^{\sigma-\gamma}(t) \geq 0 .
$$

Consider a function

$$
\begin{aligned}
\mathfrak{F}(x, t) & =\frac{1}{\mu^{\beta} \Gamma(\beta)} \exp \left[\frac{\mu-1}{\mu}(x-t)\right](x-t)^{\beta-1} \\
& =\frac{1}{\mu^{\beta} \Gamma(\beta)}(x-t)^{\beta-1}\left[1+\frac{\mu-1}{\mu}(x-t)+\frac{\left(\frac{\mu-1}{\mu}(x-t)\right)^{2}}{2}+\cdots\right] .
\end{aligned}
$$

We observe that the function $\mathfrak{F}(x, t)$ remains positive for all $t \in(a, x), a<x \leq b$, as each term of the above function is positive in view of conditions stated in Theorem 2.1. Therefore, multiplying (2.3) by $\mathfrak{F}(x, t) g^{\gamma}(t)=\frac{1}{\mu^{\beta} \Gamma(\beta)} \exp \left[\frac{\mu-1}{\mu}(x-t)\right](x-t)^{\beta-1} g^{\gamma}(t), t \in(a, x)$, $a<x \leq b$, we have

$$
\begin{aligned}
\mathfrak{F}(x, t) & {\left[(\rho-a)^{\vartheta} g^{\sigma-\gamma}(t)+(t-a)^{\vartheta} g^{\sigma-\gamma}(\rho)-(\rho-a)^{\vartheta} g^{\sigma-\gamma}(\rho)-(t-a)^{\vartheta} g^{\sigma-\gamma}(t)\right] g^{\gamma}(t) } \\
= & (\rho-a)^{\vartheta} \frac{1}{\mu^{\beta} \Gamma(\beta)} \exp \left[\frac{\mu-1}{\mu}(x-t)\right](x-t)^{\beta-1} g^{\gamma}(t)(t) g^{\sigma-\gamma}(t) \\
& +(t-a)^{\vartheta} \frac{1}{\mu^{\beta} \Gamma(\beta)} \exp \left[\frac{\mu-1}{\mu}(x-t)\right](x-t)^{\beta-1} g^{\gamma}(t) g^{\sigma-\gamma}(\rho) \\
& -(\rho-a)^{\vartheta} \frac{1}{\mu^{\beta} \Gamma(\beta)} \exp \left[\frac{\mu-1}{\mu}(x-t)\right](x-t)^{\beta-1} g^{\gamma}(t) g^{\sigma-\gamma}(\rho) \\
& -(t-a)^{\vartheta} \frac{1}{\mu^{\beta} \Gamma(\beta)} \exp \left[\frac{\mu-1}{\mu}(x-t)\right](x-t)^{\beta-1} g^{\gamma}(t) g^{\sigma-\gamma}(t) \geq 0 .
\end{aligned}
$$

Integrating (2.5) with respect to $t$ over $(a, x)$, we have

$$
\begin{aligned}
& (\rho-a)^{\vartheta} \frac{1}{\mu^{\beta} \Gamma(\beta)} \int_{a}^{x} \exp \left[\frac{\mu-1}{\mu}(x-t)\right](x-t)^{\beta-1} g^{\sigma}(t) d t \\
& \quad+g^{\sigma-\gamma}(\rho) \frac{1}{\mu^{\beta} \Gamma(\beta)} \int_{a}^{x} \exp \left[\frac{\mu-1}{\mu}(x-t)\right](x-t)^{\beta-1}(t-a)^{\vartheta} g^{\gamma}(t) d t \\
& \quad-(\rho-a)^{\vartheta} g^{\sigma-\gamma}(\rho) \frac{1}{\mu^{\beta} \Gamma(\beta)} \int_{a}^{x} \exp \left[\frac{\mu-1}{\mu}(x-t)\right](x-t)^{\beta-1} g^{\gamma}(t) d t \\
& -\frac{1}{\mu^{\beta} \Gamma(\beta)} \int_{a}^{x} \exp \left[\frac{\mu-1}{\mu}(x-t)\right](x-t)^{\beta-1}(t-a)^{\vartheta} g^{\sigma}(t) d t \geq 0 .
\end{aligned}
$$


It follows that

$$
\begin{aligned}
& (\rho-a)^{\vartheta}{ }_{a} \mathfrak{I}^{\beta, \mu}\left[g^{\sigma}(x)\right]+g^{\sigma-\gamma}(\rho)_{a} \mathfrak{I}^{\beta, \mu}\left[(x-a)^{\vartheta} g^{\gamma}(x)\right] \\
& -(\rho-a)^{\vartheta} g^{\sigma-\gamma}(\rho)_{a} \mathfrak{I}^{\beta, \mu}\left[g^{\gamma}(x)\right]-{ }_{a} \mathfrak{I}^{\beta, \mu}\left[(x-a)^{\vartheta} g^{\sigma}(x)\right] .
\end{aligned}
$$

Multiplying (2.7) by $\mathfrak{F}(x, \rho) g^{\gamma}(\rho)=\frac{1}{\mu^{\beta} \Gamma(\beta)} \exp \left[\frac{\mu-1}{\mu}(x-\rho)\right](x-\rho)^{\beta-1} g^{\gamma}(\rho), \rho \in(a, x), a<x \leq$ $b$, and integrating the resultant identity with respect to $\rho$ over $(a, x)$, we get

$$
{ }_{a} \mathfrak{I}^{\beta, \mu}\left[g^{\sigma}(x)\right]_{a} \mathfrak{I}^{\beta, \mu}\left[(x-a)^{\vartheta} g^{\gamma}(x)\right]-{ }_{a} \mathfrak{I}^{\beta, \mu}\left[(x-a)^{\vartheta} g^{\sigma}(x)\right]{ }_{a} \mathfrak{I}^{\beta, \mu}\left[g^{\gamma}(x)\right] \geq 0
$$

It follows that

$$
{ }_{a} \mathfrak{I}^{\beta, \mu}\left[g^{\sigma}(x)\right]_{a} \mathfrak{I}^{\beta, \mu}\left[(x-a)^{\vartheta} g^{\gamma}(x)\right] \geq{ }_{a} \mathfrak{I}^{\beta, \mu}\left[(x-a)^{\vartheta} g^{\sigma}(x)\right]_{a} \mathfrak{I}^{\beta, \mu}\left[g^{\gamma}(x)\right] .
$$

Dividing the above equation by ${ }_{a} \mathfrak{I}^{\beta, \mu}\left[(x-a)^{\vartheta} g^{\gamma}(x)\right]_{a} \mathfrak{I}^{\beta, \mu}\left[g^{\gamma}(x)\right]$, we get the desired inequality (2.1).

Remark The inequality in Theorem 2.1 will reverse if $g$ is an increasing function on the interval $[a, b]$.

Theorem 2.2 Let $g$ be a positive continuous and decreasing function on the interval $[a, b]$. Let $a<x \leq b, \vartheta>0, \sigma \geq \gamma>0$. Then, for generalized proportional fractional integral (1.1), we have

$$
\frac{{ }_{a} \mathfrak{I}^{\beta, \mu}\left[g^{\sigma}(x)\right]_{a} \mathfrak{I}^{\lambda, \mu}\left[(x-a)^{\vartheta} g^{\gamma}(x)\right]+{ }_{a} \mathfrak{I}^{\lambda, \mu}\left[g^{\sigma}(x)\right]_{a} \mathfrak{I}^{\beta, \mu}\left[(x-a)^{\vartheta} g^{\gamma}(x)\right]}{{ }_{a} \mathfrak{I}^{\beta, \mu}\left[(x-a)^{\vartheta} g^{\sigma}(x)\right]_{a} \mathfrak{I}^{\lambda, \mu}\left[g^{\gamma}(x)\right]+{ }_{a} \mathfrak{I}^{\lambda, \mu}\left[(x-a)^{\vartheta} g^{\sigma}(x)\right]_{a} \mathfrak{I}^{\beta, \mu}\left[g^{\gamma}(x)\right]} \geq 1,
$$

where $\mu \in(0,1], \beta, \lambda \in \mathbb{C}, \mathfrak{R}(\beta)>0$, and $\mathfrak{R}(\lambda)>0$.

Proof By multiplying both sides of (2.7) by $\mathfrak{F}(x, \rho) g^{\gamma}(\rho)=\frac{1}{\mu^{\lambda} \Gamma(\lambda)} \exp \left[\frac{\mu-1}{\mu}(x-\rho)\right](x-$ $\rho)^{\lambda-1} g^{\gamma}(\rho), \rho \in(a, x), a<x \leq b$, and integrating the resultant identity with respect to $\rho$ over $(a, x)$, we have

$$
\begin{aligned}
& { }_{a} \mathfrak{I}^{\beta, \mu}\left[g^{\sigma}(x)\right] \mathfrak{I}^{\lambda, \mu}\left[(x-a)^{\vartheta} g^{\gamma}(x)\right]+{ }_{a} \mathfrak{I}^{\lambda, \mu}\left[g^{\sigma}(x)\right]_{a} \mathfrak{I}^{\beta, \mu}\left[(x-a)^{\vartheta} g^{\gamma}(x)\right] \\
& \quad-{ }_{a} \mathfrak{I}^{\beta, \mu}\left[(x-a)^{\vartheta} g^{\sigma}(x)\right]_{a} \mathfrak{I}^{\lambda, \mu}\left[g^{\gamma}(x)\right]-{ }_{a} \mathfrak{I}^{\lambda, \mu}\left[(x-a)^{\vartheta} g^{\sigma}(x)\right]_{a} \mathfrak{I}^{\beta, \mu}\left[g^{\gamma}(x)\right] \geq 0 .
\end{aligned}
$$

Hence, dividing (2.9) by

$$
{ }_{a} \mathfrak{I}^{\beta, \mu}\left[(x-a)^{\vartheta} g^{\sigma}(x)\right]_{a} \mathfrak{I}^{\lambda, \mu}\left[g^{\gamma}(x)\right]+{ }_{a} \mathfrak{I}^{\lambda, \mu}\left[(x-a)^{\vartheta} g^{\sigma}(x)\right]_{a} \mathfrak{I}^{\beta, \mu}\left[g^{\gamma}(x)\right]
$$

completes the desired proof.

Remark Applying Theorem 2.2 for $\beta=\lambda$, we get Theorem 2.1.

Theorem 2.3 Let $g$ and $h$ be positive continuous functions on the interval $[a, b]$ such that $h$ is increasing and $g$ is decreasing function on the interval $[a, b]$. Let $a<x \leq b, \vartheta>0, \sigma \geq$ 
$\gamma>0$. Then, for generalized proportional fractional integral (1.1), we have

$$
\frac{{ }_{a} \mathfrak{I}^{\beta, \mu}\left[g^{\sigma}(x)\right]_{a} \mathfrak{I}^{\beta, \mu}\left[h^{\vartheta}(x) g^{\gamma}(x)\right]}{{ }_{a} \mathfrak{I}^{\beta, \mu}\left[h^{\vartheta}(x) g^{\sigma}(x)\right]_{a} \mathfrak{I}^{\beta, \mu}\left[g^{\gamma}(x)\right]} \geq 1,
$$

where $\mu \in(0,1], \beta \in \mathbb{C}$, and $\mathfrak{R}(\beta)>0$.

Proof Under the conditions stated in Theorem 2.3, we can write

$$
\left(h^{\vartheta}(\rho)-h^{\vartheta}(t)\right)\left(g^{\sigma-\gamma}(t)-g^{\sigma-\gamma}(\rho)\right) \geq 0,
$$

where $a<x \leq b, \vartheta>0, \sigma \geq \gamma>0$, and $t, \rho \in[a, x]$.

From (2.11), we have

$$
h^{\vartheta}(\rho) g^{\sigma-\gamma}(t)+h^{\vartheta}(t) g^{\sigma-\gamma}(\rho)-h^{\vartheta}(\rho) g^{\sigma-\gamma}(\rho)-h^{\vartheta}(t) g^{\sigma-\gamma}(t) \geq 0
$$

Multiplying both sides of (2.12) by $\mathfrak{F}(x, t) g^{\gamma}(t)=\frac{1}{\mu^{\beta} \Gamma(\beta)} \exp \left[\frac{\mu-1}{\mu}(x-t)\right](x-t)^{\beta-1} g^{\gamma}(t), t \in$ $(a, x), a<x \leq b$, where $\mathfrak{F}(x, t)$ is defined by $(2.4)$, we have

$$
\begin{aligned}
\mathfrak{F}(x, t) g^{\gamma}(t)\left[h^{\vartheta}(\rho) g^{\sigma-\gamma}(t)+h^{\vartheta}(t) g^{\sigma-\gamma}(\rho)-h^{\vartheta}(\rho) g^{\sigma-\gamma}(\rho)-h^{\vartheta}(t) g^{\sigma-\gamma}(t)\right] \\
=h^{\vartheta}(\rho) \frac{1}{\mu^{\beta} \Gamma(\beta)} \exp \left[\frac{\mu-1}{\mu}(x-t)\right](x-t)^{\beta-1} g^{\sigma}(t) \\
+h^{\vartheta}(t) \frac{1}{\mu^{\beta} \Gamma(\beta)} \exp \left[\frac{\mu-1}{\mu}(x-t)\right](x-t)^{\beta-1} g^{\sigma-\gamma}(\rho) g^{\sigma}(t) \\
\quad-h^{\vartheta}(\rho) \frac{1}{\mu^{\beta} \Gamma(\beta)} \exp \left[\frac{\mu-1}{\mu}(x-t)\right](x-t)^{\beta-1} g^{\sigma-\gamma}(\rho) g^{\sigma}(t) \\
-h^{\vartheta}(t)^{\vartheta} \frac{1}{\mu^{\beta} \Gamma(\beta)} \exp \left[\frac{\mu-1}{\mu}(x-t)\right](x-t)^{\beta-1} g^{\sigma}(t) \geq 0 .
\end{aligned}
$$

Integrating (2.13) with respect to $t$ over $(a, x)$, we have

$$
\begin{aligned}
& h^{\vartheta}(\rho) \frac{1}{\mu^{\beta} \Gamma(\beta)} \int_{a}^{x} \exp \left[\frac{\mu-1}{\mu}(x-t)\right](x-t)^{\beta-1} g^{\sigma}(t) d t \\
& \quad+g_{p}^{\sigma-\gamma_{p}}(\rho) \frac{1}{\mu^{\beta} \Gamma(\beta)} \int_{a}^{x} \exp \left[\frac{\mu-1}{\mu}(x-t)\right](x-t)^{\beta-1} h^{\vartheta}(t) g^{\gamma}(t) d t \\
& \quad-h^{\vartheta}(\rho) g^{\sigma-\gamma}(\rho) \frac{1}{\mu^{\beta} \Gamma(\beta)} \int_{a}^{x} \exp \left[\frac{\mu-1}{\mu}(x-t)\right](x-t)^{\beta-1} g^{\gamma}(t) d t \\
& -\frac{1}{\mu^{\beta} \Gamma(\beta)} \int_{a}^{x} \exp \left[\frac{\mu-1}{\mu}(x-t)\right](x-t)^{\beta-1} h^{\vartheta}(t) g^{\sigma}(t) d t \geq 0 .
\end{aligned}
$$

From (2.14), it can be written as

$$
\begin{aligned}
& h^{\vartheta}(\rho)_{a} \mathfrak{I}^{\beta, \mu}\left[g^{\sigma}(x)\right]+g^{\sigma-\gamma}(\rho)_{a} \mathfrak{I}^{\beta, \mu}\left[h^{\vartheta}(x) g^{\gamma}(x)\right] \\
& \quad-h^{\vartheta}(\rho) g^{\sigma-\gamma}(\rho)_{a} \mathfrak{I}^{\beta, \mu}\left[g^{\gamma}(x)\right]-{ }_{a} \mathfrak{I}^{\beta, \mu}\left[h^{\vartheta}(x) g^{\gamma}(x)\right] \geq 0 .
\end{aligned}
$$


Again, multiplying (2.15) by $\mathfrak{F}(x, \rho) g^{\gamma}(\rho)=\frac{1}{\mu^{\beta} \Gamma(\beta)} \exp \left[\frac{\mu-1}{\mu}(x-\rho)\right](x-\rho)^{\beta-1} g^{\gamma}(\rho), \rho \in(a, x)$, $a<x \leq b$, where $\mathfrak{F}(x, \rho)$ is defined by (2.4), and integrating the resultant identity with respect to $\rho$ over $(a, x)$, we get

$$
\begin{aligned}
& { }_{a} \mathfrak{I}^{\beta, \mu}\left[g^{\sigma}(x)\right]_{a} \mathfrak{I}^{\beta, \mu}\left[h^{\vartheta}(x) g^{\gamma}(x)\right] \\
& \quad-{ }_{a} \mathfrak{I}^{\beta, \mu}\left[h^{\vartheta}(x) g^{\sigma}(x)\right]_{a} \mathfrak{I}^{\beta, \mu}\left[g^{\gamma}(x)\right] \geq 0,
\end{aligned}
$$

which completes the desired inequality (2.10) of Theorem 2.3.

Theorem 2.4 Let $g$ and $h$ be positive continuous functions on the interval $[a, b]$ such that $h$ is increasing and $g$ is decreasing function on the interval $[a, b]$. Let $a<x \leq b, \vartheta>0, \sigma \geq$ $\gamma>0$. Then, for generalized proportional fractional integral (1.1), we have

$$
\frac{{ }_{a} \mathfrak{I}^{\beta, \mu}\left[g^{\sigma}(x)\right]_{a} \mathfrak{I}^{\lambda, \mu}\left[h^{\vartheta}(x) g^{\gamma}(x)\right]+{ }_{a} \mathfrak{I}^{\lambda, \mu}\left[g^{\sigma}(x)\right]_{a} \mathfrak{I}^{\beta, \mu}\left[h^{\vartheta}(x) g^{\gamma}(x)\right]}{{ }_{a} \mathfrak{I}^{\beta, \mu}\left[h^{\vartheta}(x) g^{\sigma}(x)\right]_{a} \mathfrak{I}^{\lambda, \mu}\left[g^{\gamma}(x)\right]+{ }_{a} \mathfrak{I}^{\lambda, \mu}\left[h^{\vartheta}(x) g^{\sigma}(x)\right]_{a} \Im^{\beta, \mu}\left[g^{\gamma}(x)\right]} \geq 1,
$$

where $\mu \in(0,1], \beta, \lambda \in \mathbb{C}, \Re(\beta)>0$, and $\Re(\lambda)>0$.

Proof Multiplying (2.15) by $\mathfrak{F}(x, \rho) g^{\gamma}(\rho)=\frac{1}{\mu^{\lambda} \Gamma(\lambda)} \exp \left[\frac{\mu-1}{\mu}(x-\rho)\right](x-\rho)^{\lambda-1} g^{\gamma}(\rho), \rho \in(a, x)$, $a<x \leq b$, where $\mathfrak{F}(x, \rho)$ is defined by (2.4), and integrating the resultant identity with respect to $\rho$ over $(a, x)$, we get

$$
\begin{aligned}
& { }_{a} \mathfrak{I}^{\beta, \mu}\left[g^{\sigma}(x)\right]_{a} \mathfrak{I}^{\lambda, \mu}\left[h^{\vartheta}(x) g^{\gamma}(x)\right]+{ }_{a} \mathfrak{I}^{\lambda, \mu}\left[g^{\sigma}(x)\right]_{a} \mathfrak{I}^{\beta, \mu}\left[h^{\vartheta}(x) g^{\gamma}(x)\right] \\
& \quad-{ }_{a} \mathfrak{I}^{\beta, \mu}\left[h^{\vartheta}(x) g^{\sigma}(x)\right]_{a} \mathfrak{I}^{\lambda, \mu}\left[g^{\gamma}(x)\right]-{ }_{a} \mathfrak{I}^{\lambda, \mu}\left[h^{\vartheta}(x) g^{\sigma}(x)\right]_{a} \mathfrak{I}^{\beta, \mu}\left[g^{\gamma}(x)\right] \geq 0 .
\end{aligned}
$$

It follows that

$$
\begin{aligned}
& \left.{ }_{a} \mathfrak{I}^{\beta, \mu}\left[g^{\sigma}(x)\right]\right]_{a} \mathfrak{I}^{\lambda, \mu}\left[h^{\vartheta}(x) g^{\gamma}(x)\right]+{ }_{a} \mathfrak{I}^{\lambda, \mu}\left[g^{\sigma}(x)\right]_{a} \mathfrak{I}^{\beta, \mu}\left[h^{\vartheta}(x) g^{\gamma}(x)\right] \\
& \quad \geq{ }_{a} \mathfrak{I}^{\beta, \mu}\left[h^{\vartheta}(x) g^{\sigma}(x)\right]_{a} \mathfrak{I}^{\lambda, \mu}\left[g^{\gamma}(x)\right]+{ }_{a} \mathfrak{I}^{\lambda, \mu}\left[h^{\vartheta}(x) g^{\sigma}(x)\right]_{a} \mathfrak{I}^{\beta, \mu}\left[g^{\gamma}(x)\right] .
\end{aligned}
$$

Dividing both sides by

$$
{ }_{a} \mathfrak{I}^{\beta, \mu}\left[h^{\vartheta}(x) g^{\sigma}(x)\right]_{a} \mathfrak{I}^{\lambda, \mu}\left[g^{\gamma}(x)\right]+{ }_{a} \Im^{\lambda, \mu}\left[h^{\vartheta}(x) g^{\sigma}(x)\right]_{a} \Im^{\beta, \mu}\left[g^{\gamma}(x)\right]
$$

gives the desired inequality (2.32).

Remark Applying Theorem 2.4 for $\beta=\lambda$, we get Theorem 2.3.

Now, we use the left generalized proportional fractional integral operator to establish some inequalities for a class of $n$-decreasing positive functions.

Theorem 2.5 Let $\left(g_{j}\right)_{j=1,2,3, \ldots, n}$ be $n$ positive continuous and decreasing functions on the interval $[a, b]$. Let $a<x \leq b, \vartheta>0, \sigma \geq \gamma_{p}>0$ for any fixed $p \in\{1,2,3, \ldots, n\}$. Then, for generalized proportional fractional integral operator (1.1), we have

$$
\frac{{ }_{a} \mathfrak{I}^{\beta, \mu}\left[\prod_{j \neq p}^{n} g_{j}^{\gamma_{j}} g_{p}^{\sigma}(x)\right]}{{ }_{a} \mathfrak{I}^{\beta, \mu}\left[\prod_{j=1}^{n} g_{j}^{\gamma_{j}}(x)\right]} \geq \frac{\mathfrak{a}^{\beta, \mu}\left[(x-a)^{\vartheta} \prod_{j \neq p}^{n} g_{j}^{\gamma_{j}} g_{p}^{\sigma}(x)\right]}{{ }_{a} \mathfrak{I}^{\beta, \mu}\left[(x-a)^{\vartheta} \prod_{j=1}^{n} g_{j}^{\gamma_{j}}(x)\right]},
$$

where $\mu \in(0,1], \beta \in \mathbb{C}$, and $\Re(\beta)>0$. 
Proof Since $\left(g_{j}\right)_{j=1,2,3, \ldots, n}$ are $n$ positive continuous and decreasing functions on the interval $[a, b]$, we have

$$
\left((\rho-a)^{\vartheta}-(t-a)^{\vartheta}\right)\left(g_{p}^{\sigma-\gamma_{p}}(t)-g_{p}^{\sigma-\gamma_{p}}(\rho)\right) \geq 0
$$

for any fixed $p \in\{1,2,3, \ldots, n\}, a<x \leq b, \vartheta>0, \sigma \geq \gamma_{p}>0$, and $t, \rho \in[a, x]$. By (2.18), we have

$$
(\rho-a)^{\vartheta} g_{p}^{\sigma-\gamma_{p}}(t)+(t-a)^{\vartheta} g_{p}^{\sigma-\gamma_{p}}(\rho) \geq(\rho-a)^{\vartheta} g_{p}^{\sigma-\gamma_{p}}(\rho)+(t-a)^{\vartheta} g_{p}^{\sigma-\gamma_{p}}(t) .
$$

Multiplying both sides of (2.19) by $\mathfrak{F}(x, t) \prod_{j=1}^{n} g_{j}^{\gamma_{j}}(t)=\frac{1}{\mu^{\beta} \Gamma(\beta)} \exp \left[\frac{\mu-1}{\mu}(x-t)\right](x-t)^{\beta-1} \times$ $\prod_{j=1}^{n} g_{j}^{\gamma_{j}}(t), t \in(a, x), a<x \leq b$, where $\mathfrak{F}(x, t)$ is defined by (2.4), we have

$$
\begin{aligned}
& \mathfrak{F}(x, t) {\left[(\rho-a)^{\vartheta} g^{\sigma-\gamma}(t)+(t-a)^{\vartheta} g^{\sigma-\gamma}(\rho)-(\rho-a)^{\vartheta} g^{\sigma-\gamma}(\rho)-(t-a)^{\vartheta} g^{\sigma-\gamma}(t)\right] \prod_{j=1}^{n} g_{j}^{\gamma_{j}}(t) } \\
&=(\rho-a)^{\vartheta} \frac{1}{\mu^{\beta} \Gamma(\beta)} \exp \left[\frac{\mu-1}{\mu}(x-t)\right](x-t)^{\beta-1} \prod_{j=1}^{n} g_{j}^{\gamma_{j}}(t) g_{p}^{\sigma-\gamma_{p}}(t) \\
&+(t-a)^{\vartheta} \frac{1}{\mu^{\beta} \Gamma(\beta)} \exp \left[\frac{\mu-1}{\mu}(x-t)\right](x-t)^{\beta-1} \prod_{j=1}^{n} g_{j}^{\gamma_{j}}(t) g_{p}^{\sigma-\gamma_{p}}(\rho) \\
& \geq(\rho-a)^{\vartheta} \frac{1}{\mu^{\beta} \Gamma(\beta)} \exp \left[\frac{\mu-1}{\mu}(x-t)\right](x-t)^{\beta-1} \prod_{j=1}^{n} g_{j}^{\gamma_{j}}(t) g_{p}^{\sigma-\gamma_{p}}(\rho) \\
& \quad+(t-a)^{\vartheta} \frac{1}{\mu^{\beta} \Gamma(\beta)} \exp \left[\frac{\mu-1}{\mu}(x-t)\right](x-t)^{\beta-1} \prod_{j=1}^{n} g_{j}^{\gamma_{j}}(t) g_{p}^{\sigma-\gamma_{p}}(t)
\end{aligned}
$$

Integrating (2.20) with respect to $t$ over $(a, x)$, we have

$$
\begin{aligned}
& (\rho-a)^{\vartheta} \frac{1}{\mu^{\beta} \Gamma(\beta)} \int_{a}^{x} \exp \left[\frac{\mu-1}{\mu}(x-t)\right](x-t)^{\beta-1} \prod_{j=1}^{n} g_{j}^{\gamma_{j}}(t) g_{p}^{\sigma-\gamma_{p}}(t) d t \\
& \quad+g_{p}^{\sigma-\gamma_{p}}(\rho) \frac{1}{\mu^{\beta} \Gamma(\beta)} \int_{a}^{x} \exp \left[\frac{\mu-1}{\mu}(x-t)\right](x-t)^{\beta-1}(t-a)^{\vartheta} \prod_{j=1}^{n} g_{j}^{\gamma_{j}}(t) d t \\
& \geq(\rho-a)^{\vartheta} g_{p}^{\sigma-\gamma_{p}}(\rho) \frac{1}{\mu^{\beta} \Gamma(\beta)} \int_{a}^{x} \exp \left[\frac{\mu-1}{\mu}(x-t)\right](x-t)^{\beta-1} \prod_{j=1}^{n} g_{j}^{\gamma_{j}}(t) d t \\
& +\frac{1}{\mu^{\beta} \Gamma(\beta)} \int_{a}^{x} \exp \left[\frac{\mu-1}{\mu}(x-t)\right](x-t)^{\beta-1}(t-a)^{\vartheta} \prod_{j=1}^{n} g_{j}^{\gamma_{j}}(t) g_{p}^{\sigma-\gamma_{p}}(t) d t .
\end{aligned}
$$

From (2.21), it follows that

$$
\begin{aligned}
& (\rho-a)^{\vartheta}{ }_{a} \mathfrak{I}^{\beta, \mu}\left[\prod_{j \neq p}^{n} g_{j}^{\gamma_{j}} g_{p}^{\sigma}(x)\right]+g_{p}^{\sigma-\gamma_{p}}(\rho)_{a} \mathfrak{I}^{\beta, \mu}\left[(x-a)^{\vartheta} \prod_{j=1}^{n} g_{j}^{\gamma_{j}}(x)\right] \\
& \geq(\rho-a)^{\vartheta} g_{p}^{\sigma-\gamma_{p}}(\rho)_{a} \mathfrak{I}^{\beta, \mu}\left[\prod_{j=1}^{n} g_{j}^{\gamma_{j}}(x)\right]-{ }_{a} \mathfrak{I}^{\beta, \mu}\left[(x-a)^{\vartheta} \prod_{j \neq p}^{n} g_{j}^{\gamma_{j}} g_{p}^{\sigma}(x)\right] .
\end{aligned}
$$


Again, multiplying both sides of $(2.22)$ by $\mathfrak{F}(x, \rho) \prod_{j=1}^{n} g_{j}^{\gamma_{j}}(\rho)=\frac{1}{\mu^{\beta} \Gamma(\beta)} \exp \left[\frac{\mu-1}{\mu}(x-\rho)\right](x-$ $\rho)^{\beta-1} \prod_{j=1}^{n} g_{j}^{\gamma_{j}}(\rho), \rho \in(a, x), a<x \leq b$, where $\mathfrak{F}(x, \rho)$ is defined by (2.4), and integrating the resultant identity with respect to $\rho$ over $(a, x)$, we get

$$
\begin{aligned}
a_{a} \mathfrak{I}^{\beta, \mu} & {\left[\prod_{j \neq p}^{n} g_{j}^{\gamma_{j}} g_{p}^{\sigma}(x)\right] \mathfrak{I}^{\beta, \mu}\left[(x-a)^{\vartheta} \prod_{j=1}^{n} g_{j}^{\gamma_{j}}(x)\right] } \\
\geq & \mathfrak{a}^{\beta, \mu}\left[(x-a)^{\vartheta} \prod_{j \neq p}^{n} g_{j}^{\gamma_{j}} g_{p}^{\sigma}(x)\right]{ }_{a} \mathfrak{I}^{\beta, \mu}\left[\prod_{j=1}^{n} g_{j}^{\gamma_{j}}(x)\right],
\end{aligned}
$$

which completes the desired inequality (2.17).

Remark The inequality in Theorem 2.5 will reverse if $\left(g_{j}\right)_{j=1,2,3, \ldots, n}$ are increasing functions on the interval $[a, b]$.

Theorem 2.6 Let $\left(g_{j}\right)_{j=1,2,3, \ldots, n}$ be $n$ positive continuous and decreasing functions on the interval $[a, b]$. Let $a<x \leq b, \vartheta>0, \sigma \geq \gamma_{p}>0$ for any fixed $p \in\{1,2,3, \ldots, n\}$. Then, for generalized proportional fractional integral (1.1), we have

$$
\begin{aligned}
& \left(a^{\mathfrak{I}^{\beta, \mu}}\left[\prod_{j \neq p}^{n} g_{j}^{\gamma_{j}} g_{p}^{\sigma}(x)\right] a_{a} \mathfrak{I}^{\lambda, \mu}\left[(x-a)^{\vartheta} \prod_{j=1}^{n} g_{j}^{\gamma_{j}}(x)\right]\right. \\
& \left.+{ }_{a} \mathfrak{I}^{\lambda, \mu}\left[\prod_{j \neq p}^{n} g_{j}^{\gamma_{j}} g_{p}^{\sigma}(x)\right] a_{a} \mathfrak{I}^{\beta, \mu}\left[(x-a)^{\vartheta} \prod_{j=1}^{n} g_{j}^{\gamma_{j}}(x)\right]\right) \\
& \quad /\left(a_{a} \mathfrak{I}^{\beta, \mu}\left[(x-a)^{\vartheta} \prod_{j \neq p}^{n} g_{j}^{\gamma_{j}} g_{p}^{\sigma}(x)\right] \mathfrak{I}^{\lambda, \mu}\left[\prod_{j=1}^{n} g_{j}^{\gamma_{j}}(x)\right]\right. \\
& \left.\quad+{ }_{a} \mathfrak{I}^{\lambda, \mu}\left[(x-a)^{\vartheta} \prod_{j \neq p}^{n} g_{j}^{\gamma_{j}} g_{p}^{\sigma}(x)\right] a^{\mathfrak{I}^{\beta, \mu}}\left[\prod_{j=1}^{n} g_{j}^{\gamma_{j}}(x)\right]\right) \geq 1,
\end{aligned}
$$

where $\mu \in(0,1], \beta, \lambda \in \mathbb{C}, \mathfrak{R}(\beta)>0$, and $\mathfrak{R}(\lambda)>0$.

Proof Multiplying both sides of (2.22) by $\mathfrak{F}(x, \rho) \prod_{j=1}^{n} g_{j}^{\gamma_{j}}(\rho)=\frac{1}{\mu^{\lambda} \Gamma(\lambda)} \exp \left[\frac{\mu-1}{\mu}(x-\rho)\right](x-$ $\rho)^{\lambda-1} \prod_{j=1}^{n} g_{j}^{\gamma_{j}}(\rho), \rho \in(a, x), a<x \leq b$, where $\mathfrak{F}(x, \rho)$ is defined by (2.4), and integrating the resultant identity with respect to $\rho$ over $(a, x)$, we get

$$
\begin{aligned}
a_{a} \mathfrak{I}^{\beta, \mu} & {\left[\prod_{j \neq p}^{n} g_{j}^{\gamma_{j}} g_{p}^{\sigma}(x)\right] a_{a} \mathfrak{I}^{\lambda, \mu}\left[(x-a)^{\vartheta} \prod_{j=1}^{n} g_{j}^{\gamma_{j}}(x)\right] } \\
& +a_{a} \mathfrak{I}^{\lambda, \mu}\left[\prod_{j \neq p}^{n} g_{j}^{\gamma_{j}} g_{p}^{\sigma}(x)\right] \mathfrak{I}^{\beta, \mu}\left[(x-a)^{\vartheta} \prod_{j=1}^{n} g_{j}^{\gamma_{j}}(x)\right] \\
\geq & a_{a} \mathfrak{I}^{\beta, \mu}\left[(x-a)^{\vartheta} \prod_{j \neq p}^{n} g_{j}^{\gamma_{j}} g_{p}^{\sigma}(x)\right] \mathfrak{a}^{\lambda, \mu}\left[\prod_{j=1}^{n} g_{j}^{\gamma_{j}}(x)\right] \\
& +{ }_{a} \mathfrak{I}^{\lambda, \mu}\left[(x-a)^{\vartheta} \prod_{j \neq p}^{n} g_{j}^{\gamma_{j}} g_{p}^{\sigma}(x)\right] a^{\mathfrak{I}^{\beta, \mu}}\left[\prod_{j=1}^{n} g_{j}^{\gamma_{j}}(x)\right] .
\end{aligned}
$$


It follows that

$$
\begin{aligned}
a_{a} \mathfrak{I}^{\beta, \mu} & {\left[\prod_{j \neq p}^{n} g_{j}^{\gamma_{j}} g_{p}^{\sigma}(x)\right] \mathfrak{I}^{\lambda^{\lambda, \mu}}\left[(x-a)^{\vartheta} \prod_{j=1}^{n} g_{j}^{\gamma_{j}}(x)\right] } \\
& +{ }_{a} \mathfrak{I}^{\lambda, \mu}\left[\prod_{j \neq p}^{n} g_{j}^{\gamma_{j}} g_{p}^{\sigma}(x)\right] \mathfrak{I}^{\beta, \mu}\left[(x-a)^{\vartheta} \prod_{j=1}^{n} g_{j}^{\gamma_{j}}(x)\right] \\
\geq & { }_{a} \mathfrak{I}^{\beta, \mu}\left[(x-a)^{\vartheta} \prod_{j \neq p}^{n} g_{j}^{\gamma_{j}} g_{p}^{\sigma}(x)\right] \mathfrak{I}^{\lambda, \mu}\left[\prod_{j=1}^{n} g_{j}^{\gamma_{j}}(x)\right] \\
& +{ }_{a} \mathfrak{I}^{\lambda, \mu}\left[(x-a)^{\vartheta} \prod_{j \neq p}^{n} g_{j}^{\gamma_{j}} g_{p}^{\sigma}(x)\right] \mathfrak{I}^{\beta, \mu}\left[\prod_{j=1}^{n} g_{j}^{\gamma_{j}}(x)\right] .
\end{aligned}
$$

Hence, dividing (2.25) by

$$
\begin{aligned}
& a_{a} \mathfrak{I}^{\beta, \mu}\left[(x-a)^{\vartheta} \prod_{j \neq p}^{n} g_{j}^{\gamma_{j}} g_{p}^{\sigma}(x)\right] a \mathfrak{I}^{\lambda, \mu}\left[\prod_{j=1}^{n} g_{j}^{\gamma_{j}}(x)\right] \\
& +{ }_{a} \mathfrak{I}^{\lambda, \mu}\left[(x-a)^{\vartheta} \prod_{j \neq p}^{n} g_{j}^{\gamma_{j}} g_{p}^{\sigma}(x)\right] \mathfrak{I}^{\beta, \mu}\left[\prod_{j=1}^{n} g_{j}^{\gamma_{j}}(x)\right]
\end{aligned}
$$

completes the desired proof.

Remark Applying Theorem 2.6 for $\beta=\lambda$, we get Theorem 2.5.

Theorem 2.7 Let $\left(g_{j}\right)_{j=1,2,3, \ldots, n}$ and $h$ be positive continuous functions on the interval $[a, b]$ such that $h$ is increasing and $\left(g_{j}\right)_{j=1,2,3, \ldots, n}$ are decreasing functions on the interval $[a, b]$. Let $a<x \leq b, \vartheta>0, \sigma \geq \gamma_{p}>0$ for any fixed $p \in\{1,2,3, \ldots, n\}$. Then, for generalized proportional fractional integral (1.1), we have

$$
\frac{{ }_{a} \mathfrak{I}^{\beta, \mu}\left[\prod_{j \neq p}^{n} g_{j}^{\gamma_{j}} g_{p}^{\sigma}(x)\right]_{a} \mathfrak{I}^{\beta, \mu}\left[h^{\vartheta}(x) \prod_{j=1}^{n} g_{j}^{\gamma_{j}}(x)\right]}{{ }_{a} \mathfrak{I}^{\beta, \mu}\left[h^{\vartheta}(x) \prod_{j \neq p}^{n} g_{j}^{\gamma_{j}} g_{p}^{\sigma}(x)\right]_{a} \Im^{\beta, \mu}\left[\prod_{j=1}^{n} g_{j}^{\gamma_{j}}(x)\right]} \geq 1,
$$

where $\mu \in(0,1], \beta \in \mathbb{C}$, and $\Re(\beta)>0$.

Proof Under the conditions stated in Theorem 2.7, we can write

$$
\left(h^{\vartheta}(\rho)-h^{\vartheta}(t)\right)\left(g_{p}^{\sigma-\gamma_{p}}(t)-g_{p}^{\sigma-\gamma_{p}}(\rho)\right) \geq 0
$$

for any fixed $p \in\{1,2,3, \ldots, n\}, a<x \leq b, \vartheta>0, \sigma \geq \gamma_{p}>0$, and $t, \rho \in[a, x]$.

From (2.27), we have

$$
h^{\vartheta}(\rho) g_{p}^{\sigma-\gamma_{p}}(t)+h^{\vartheta}(t) g_{p}^{\sigma-\gamma_{p}}(\rho)-h^{\vartheta}(\rho) g_{p}^{\sigma-\gamma_{p}}(\rho)-h^{\vartheta}(t) g_{p}^{\sigma-\gamma_{p}}(t) \geq 0 \text {. }
$$


Multiplying (2.28) by $\mathfrak{F}(x, t) \prod_{j=1}^{n} g_{j}^{\gamma_{j}}(t)=\frac{1}{\mu^{\beta} \Gamma(\beta)} \exp \left[\frac{\mu-1}{\mu}(x-t)\right](x-t)^{\beta-1} \prod_{j=1}^{n} g_{j}^{\gamma_{j}}(t), t \in$ $(a, x), a<x \leq b$, where $\mathfrak{F}(x, t)$ is defined by (2.4), we have

$$
\begin{aligned}
& h^{\vartheta}(\rho) \frac{1}{\mu^{\beta} \Gamma(\beta)} \exp \left[\frac{\mu-1}{\mu}(x-t)\right](x-t)^{\beta-1} \prod_{j=1}^{n} g_{j}^{\gamma_{j}}(t) g_{p}^{\sigma-\gamma_{p}}(t) \\
& \quad+h^{\vartheta}(t) \frac{1}{\mu^{\beta} \Gamma(\beta)} \exp \left[\frac{\mu-1}{\mu}(x-t)\right](x-t)^{\beta-1} \prod_{j=1}^{n} g_{j}^{\gamma_{j}}(t) g_{p}^{\sigma-\gamma_{p}}(\rho) \\
& \quad-h^{\vartheta}(\rho) \frac{1}{\mu^{\beta} \Gamma(\beta)} \exp \left[\frac{\mu-1}{\mu}(x-t)\right](x-t)^{\beta-1} \prod_{j=1}^{n} g_{j}^{\gamma_{j}}(t) g_{p}^{\sigma-\gamma_{p}}(\rho) \\
& -h^{\vartheta}(t)^{\vartheta} \frac{1}{\mu^{\beta} \Gamma(\beta)} \exp \left[\frac{\mu-1}{\mu}(x-t)\right](x-t)^{\beta-1} \prod_{j=1}^{n} g_{j}^{\gamma_{j}}(t) g_{p}^{\sigma-\gamma_{p}}(t) \geq 0 .
\end{aligned}
$$

Integrating (2.29) with respect to $t$ over $(a, x)$, we have

$$
\begin{aligned}
& h^{\vartheta}(\rho) \frac{1}{\mu^{\beta} \Gamma(\beta)} \int_{a}^{x} \exp \left[\frac{\mu-1}{\mu}(x-t)\right](x-t)^{\beta-1} \prod_{j=1}^{n} g_{j}^{\gamma_{j}}(t) g_{p}^{\sigma-\gamma_{p}}(t) d t \\
& \quad+g_{p}^{\sigma-\gamma_{p}}(\rho) \frac{1}{\mu^{\beta} \Gamma(\beta)} \int_{a}^{x} \exp \left[\frac{\mu-1}{\mu}(x-t)\right](x-t)^{\beta-1} h^{\vartheta}(t)^{\vartheta} \prod_{j=1}^{n} g_{j}^{\gamma_{j}}(t) d t \\
& \quad-h^{\vartheta}(\rho) g_{p}^{\sigma-\gamma_{p}}(\rho) \frac{1}{\mu^{\beta} \Gamma(\beta)} \int_{a}^{x} \exp \left[\frac{\mu-1}{\mu}(x-t)\right](x-t)^{\beta-1} \prod_{j=1}^{n} g_{j}^{\gamma_{j}}(t) d t \\
& -\frac{1}{\mu^{\beta} \Gamma(\beta)} \int_{a}^{x} \exp \left[\frac{\mu-1}{\mu}(x-t)\right](x-t)^{\beta-1} h^{\vartheta}(t) \prod_{j=1}^{n} g_{j}^{\gamma_{j}}(t) g_{p}^{\sigma-\gamma_{p}}(t) d t \geq 0 .
\end{aligned}
$$

From (2.30), we can write

$$
\begin{aligned}
& h^{\vartheta}(\rho)_{a} \mathfrak{I}^{\beta, \mu}\left[\prod_{j \neq p}^{n} g_{j}^{\gamma_{j}} g_{p}^{\sigma}(x)\right]+g_{p}^{\sigma-\gamma_{p}}(\rho)_{a} \mathfrak{I}^{\beta, \mu}\left[h^{\vartheta}(x) \prod_{j=1}^{n} g_{j}^{\gamma_{j}}(x)\right] \\
& -h^{\vartheta}(\rho) g_{p}^{\sigma-\gamma_{p}}(\rho)_{a} \mathfrak{I}^{\beta, \mu}\left[\prod_{j=1}^{n} g_{j}^{\gamma_{j}}(x)\right]-{ }_{a} \mathfrak{I}^{\beta, \mu}\left[h^{\vartheta}(x) \prod_{j \neq p}^{n} g_{j}^{\gamma_{j}} g_{p}^{\sigma}(x)\right] \geq 0 .
\end{aligned}
$$

Again, multiplying (2.31) by $\mathfrak{F}(x, \rho) \prod_{j=1}^{n} g_{j}^{\gamma_{j}}(\rho)=\frac{1}{\mu^{\beta} \Gamma(\beta)} \exp \left[\frac{\mu-1}{\mu}(x-\rho)\right](x-\rho)^{\beta-1} \times$ $\prod_{j=1}^{n} g_{j}^{\gamma j}(\rho), \rho \in(a, x), a<x \leq b$, where $\mathfrak{F}(x, \rho)$ is defined by (2.4) and integrating the resultant identity with respect to $\rho$ over $(a, x)$, we get

$$
\begin{aligned}
& { }_{a} \mathfrak{I}^{\beta, \mu}\left[\prod_{j \neq p}^{n} g_{j}^{\gamma_{j}} g_{p}^{\sigma}(x)\right] \mathfrak{I}^{\beta, \mu}\left[h^{\vartheta}(x) \prod_{j=1}^{n} g_{j}^{\gamma_{j}}(x)\right] \\
& \quad-{ }_{a} \mathfrak{I}^{\beta, \mu}\left[h^{\vartheta}(x) \prod_{j \neq p}^{n} g_{j}^{\gamma_{j}} g_{p}^{\sigma}(x)\right]{ }_{a} \mathfrak{I}^{\beta, \mu}\left[\prod_{j=1}^{n} g_{j}^{\gamma_{j}}(x)\right] \geq 0,
\end{aligned}
$$

which completes the desired inequality (2.26) of Theorem 2.7 . 
Theorem 2.8 Let $\left(g_{j}\right)_{j=1,2,3, \ldots, n}$ and $h$ be positive continuous functions on the interval $[a, b]$ such that $h$ is increasing and $\left(g_{j}\right)_{j=1,2,3, \ldots, n}$ are decreasing functions on the interval $[a, b]$. Let $a<x \leq b, \vartheta>0, \sigma \geq \gamma_{p}>0$ for any fixed $p \in\{1,2,3, \ldots, n\}$. Then, for generalized proportional fractional integral (1.1), we have

$$
\begin{aligned}
& \left(a^{\Im^{\beta, \mu}}\left[\prod_{j \neq p}^{n} g_{j}^{\gamma_{j}} g_{p}^{\sigma}(x)\right] \mathfrak{I}^{\lambda, \mu}\left[h^{\vartheta}(x) \prod_{j=1}^{n} g_{j}^{\gamma_{j}}(x)\right]\right. \\
& \left.+{ }_{a} \mathfrak{I}^{\lambda, \mu}\left[\prod_{j \neq p}^{n} g_{j}^{\gamma_{j}} g_{p}^{\sigma}(x)\right] \mathfrak{I}^{\beta, \mu}\left[h^{\vartheta}(x) \prod_{j=1}^{n} g_{j}^{\gamma_{j}}(x)\right]\right) \\
& /\left({ }_{a} \mathfrak{I}^{\beta, \mu}\left[h^{\vartheta}(x) \prod_{j \neq p}^{n} g_{j}^{\gamma_{j}} g_{p}^{\sigma}(x)\right]{ }_{a} \mathfrak{I}^{\lambda, \mu}\left[\prod_{j=1}^{n} g_{j}^{\gamma_{j}}(x)\right]\right. \\
& \left.+a_{a} \mathfrak{I}^{\lambda, \mu}\left[h^{\vartheta}(x) \prod_{j \neq p}^{n} g_{j}^{\gamma_{j}} g_{p}^{\sigma}(x)\right] \mathfrak{I}^{\beta, \mu}\left[\prod_{j=1}^{n} g_{j}^{\gamma_{j}}(x)\right]\right) \geq 1,
\end{aligned}
$$

where $\mu \in(0,1], \beta, \lambda \in \mathbb{C}, \Re(\beta)>0$, and $\Re(\lambda)>0$.

Proof Multiplying (2.31) by $\mathfrak{F}(x, \rho) \prod_{j=1}^{n} g_{j}^{\gamma_{j}}(\rho)=\frac{1}{\mu^{\lambda} \Gamma(\lambda)} \exp \left[\frac{\mu-1}{\mu}(x-\rho)\right](x-\rho)^{\lambda-1} \times$ $\prod_{j=1}^{n} g_{j}^{\gamma_{j}}(\rho), \rho \in(a, x), a<x \leq b$, where $\mathfrak{F}(x, \rho)$ is defined by (2.4), and integrating the resultant identity with respect to $\rho$ over $(a, x)$, we get

$$
\begin{aligned}
& a_{a} \mathfrak{I}^{\beta, \mu}\left[\prod_{j \neq p}^{n} g_{j}^{\gamma_{j}} g_{p}^{\sigma}(x)\right] \mathfrak{I}^{\lambda, \mu}\left[h^{\vartheta}(x) \prod_{j=1}^{n} g_{j}^{\gamma_{j}}(x)\right] \\
& +{ }_{a} \mathfrak{I}^{\lambda, \mu}\left[\prod_{j \neq p}^{n} g_{j}^{\gamma_{j}} g_{p}^{\sigma}(x)\right] \mathfrak{I}^{\beta, \mu}\left[h^{\vartheta}(x) \prod_{j=1}^{n} g_{j}^{\gamma_{j}}(x)\right] \\
& -{ }_{a} \mathfrak{I}^{\beta, \mu}\left[h^{\vartheta}(x) \prod_{j \neq p}^{n} g_{j}^{\gamma_{j}} g_{p}^{\sigma}(x)\right] \mathfrak{I}^{\lambda, \mu}\left[\prod_{j=1}^{n} g_{j}^{\gamma_{j}}(x)\right] \\
& -a_{a} \mathfrak{I}^{\lambda, \mu}\left[h^{\vartheta}(x) \prod_{j \neq p}^{n} g_{j}^{\gamma_{j}} g_{p}^{\sigma}(x)\right] \mathfrak{I}_{a}^{\beta, \mu}\left[\prod_{j=1}^{n} g_{j}^{\gamma_{j}}(x)\right] \geq 0 .
\end{aligned}
$$

It follows that

$$
\begin{aligned}
& a \Im^{\beta, \mu} {\left[\prod_{j \neq p}^{n} g_{j}^{\gamma_{j}} g_{p}^{\sigma}(x)\right] \mathfrak{I}^{\lambda, \mu}\left[h^{\vartheta}(x) \prod_{j=1}^{n} g_{j}^{\gamma_{j}}(x)\right] } \\
&+{ }_{a} \mathfrak{I}^{\lambda, \mu}\left[\prod_{j \neq p}^{n} g_{j}^{\gamma_{j}} g_{p}^{\sigma}(x)\right] \mathfrak{I}^{\beta, \mu}\left[h^{\vartheta}(x) \prod_{j=1}^{n} g_{j}^{\gamma_{j}}(x)\right] \\
& \geq{ }_{a} \mathfrak{I}^{\beta, \mu}\left[h^{\vartheta}(x) \prod_{j \neq p}^{n} g_{j}^{\gamma_{j}} g_{p}^{\sigma}(x)\right] \mathfrak{I}_{a}^{\lambda, \mu}\left[\prod_{j=1}^{n} g_{j}^{\gamma_{j}}(x)\right] \\
& \quad+{ }_{a} \mathfrak{I}^{\lambda, \mu}\left[h^{\vartheta}(x) \prod_{j \neq p}^{n} g_{j}^{\gamma_{j}} g_{p}^{\sigma}(x)\right] \mathfrak{I}^{\Im^{\beta, \mu}}\left[\prod_{j=1}^{n} g_{j}^{\gamma_{j}}(x)\right] .
\end{aligned}
$$


Dividing both sides by

$$
\begin{aligned}
& { }_{a} \mathfrak{I}^{\beta, \mu}\left[h^{\vartheta}(x) \prod_{j \neq p}^{n} g_{j}^{\gamma_{j}} g_{p}^{\sigma}(x)\right] a_{a} \mathfrak{I}^{\lambda, \mu}\left[\prod_{j=1}^{n} g_{j}^{\gamma_{j}}(x)\right] \\
& +{ }_{a} \mathfrak{I}^{\lambda, \mu}\left[h^{\vartheta}(x) \prod_{j \neq p}^{n} g_{j}^{\gamma_{j}} g_{p}^{\sigma}(x)\right]{ }_{a} \mathfrak{I}^{\beta, \mu}\left[\prod_{j=1}^{n} g_{j}^{\gamma_{j}}(x)\right]
\end{aligned}
$$

gives the desired inequality (2.32).

Remark Applying Theorem 2.8 for $\beta=\lambda$, we get Theorem 2.7.

Remark Similarly, we can establish the inequalities for the right generalized proportional fractional integral defined by (1.4).

\section{Concluding remarks}

In this present paper, we established certain inequalities by employing the left generalized proportional fractional integral operator. Also, some inequalities for a class of $n$ positive continuous and decreasing functions on the interval $[a, b]$ are presented. In [20], Liu et al. introduced interesting integral inequalities for continuous functions on $[a, b]$. Later on, Dahmani [14] generalized the work of [20] involving the Riemann-Liouville fractional integral operators. Recently, Jarad et al. [16] introduced Caputo and Riemann-Liouville generalized proportional fractional derivatives which comprise exponential functions in their kernels. They proved that the newly defined proportional fractional integrals possess a semi-group property and they provide an undeviating generalization to the existing $\mathrm{Ca}$ puto and Riemann-Liouville fractional derivatives and integrals. Therefore the inequalities obtained in this paper are the generalization of integral inequalities involving the Riemann-Liouville fractional integral operators. If we consider $\mu=1$, then the inequalities obtained in this paper will reduce to the integral inequalities involving the RiemannLiouville fractional integral operators introduced by Dahmani [14]. Some of the special cases of our main results are found in [20]. The results obtained in this paper give some contributions towards the theory of integral inequalities and fractional calculus and are expected to lead to some applications for establishing the uniqueness of solutions in generalized proportional fractional differential equations. Also, as an application, we will try to find the analytical solutions of some space-time conformable fractional differential equations using a different method in our future papers.

\section{Acknowledgements}

The second author would like to thank Prince Sultan University for funding this work through research group Nonlinear Analysis Methods in Applied Mathematics (NAMAM) group number RG-DES-2017-01-17.

\section{Funding}

Not applicable.

Availability of data and materials

Not applicable.

Competing interests

The authors declare that they have no competing interests. 


\section{Author details}

'Department of Mathematics, Shaheed Benazir Bhutto University, Sheringal, Pakistan. ${ }^{2}$ Department of Mathematics and General Sciences, Prince Sultan University, Riyadh, Saudi Arabia. ${ }^{3}$ Department of Mathematics, College of Arts and Sciences, Prince Sattam bin Abdulaziz University, Wadi Aldawser, Saudi Arabia.

\section{Publisher's Note}

Springer Nature remains neutral with regard to jurisdictional claims in published maps and institutional affiliations.

Received: 6 May 2019 Accepted: 3 September 2019 Published online: 11 September 2019

\section{References}

1. Aldhaifallah, M., Tomar, M., Nisar, K.S., Purohit, S.D.: Some new inequalities for (k, s)-fractional integrals. J. Nonlinear Sci. Appl. 9(9), 5374-5381 (2016)

2. Agarwal, P., Jleli, M., Tomar, M.: Certain Hermite-Hadamard type inequalities via generalized $k$-fractional integrals. J. Inequal. Appl. 2017, 55 (2017)

3. Abdeljawad, T.: On conformable fractional calculus. J. Comput. Appl. Math. 279, 57-66 (2015). https://doi.org/10.1016/j.cam.2014.10.016

4. Abdeljawad, T., Baleanu, D.: Monotonicity results for fractional difference operators with discrete exponential kernels. Adv. Differ. Equ. 2017, 78 (2017). https://doi.org/10.1186/s13662-017-1126-1

5. Abdeljawad, T., Baleanu, D.: On fractional derivatives with exponential kernel and their discrete versions. Rep. Math. Phys. 80, 11-27 (2017). https://doi.org/10.1016/S0034-4877(17)30059-9

6. Alzabut, J., Abdeljawad, T., Jarad, F., Sudsutad, W.: A Gronwall inequality via the generalized proportional fractional derivative with applications. J. Inequal. Appl. 2019, 101 (2019)

7. Atangana, A., Baleanu, D.: New fractional derivatives with nonlocal and non-singular kernel: theory and application to heat transfer model. Therm. Sci. 20, 763-769 (2016). https://doi.org/10.2298/TSCl160111018A

8. Anderson, D.R., Ulness, D.J.: Newly defined conformable derivatives. Adv. Dyn. Syst. Appl. 10(2), 109-137 (2015)

9. Caputo, M., Fabrizio, M.: A new definition of fractional derivative without singular kernel. Prog. Fract. Differ. Appl. 1(2), 73-85 (2015)

10. Huang, C.J., Rahman, G., Nisar, K.S., Ghaffar, A., Qi, F.: Some inequalities of Hermite-Hadamard type for k-fractional conformable integrals. Aust. J. Math. Anal. Appl. 16(1), 1-9 (2019)

11. Baleanu, D., Diethelm, K., Scalas, E.: Fractional Calculus: Models and Numerical Methods. World Scientific, Singapore (2012)

12. Dahmani, Z., Tabharit, L.: On weighted Gruss type inequalities via fractional integration. J. Adv. Res. Pure Math. 2 $31-38(2010)$

13. Dahmani, Z.: New inequalities in fractional integrals. Int. J. Nonlinear Sci. 9, 493-497 (2010)

14. Dahmani, Z:: New classes of integral inequalities of fractional order. Matematiche LXIX(1), 237-247 (2014). https://doi.org/10.4418/2014.69.1.18

15. Khan, H., Abdeljawad, T., Tunc, C., Alkhazzan, A., Khan, A.: Minkowski's inequality for the AB-fractional integral operator. J. Inequal. Appl. 2019, 96 (2019)

16. Jarad, F., Abdeljawad, T., Alzabut, J.: Generalized fractional derivatives generated by a class of local proportional derivatives. Eur. Phys. J. Spec. Top. 226, 3457-3471 (2017). https://doi.org/10.1140/epjst/e2018-00021-7

17. Khalil, R., Al Horani, M., Yousef, A., Sababheh, M.: A new definition of fractional derivative. J. Comput. Appl. Math. 264(65), 65-70 (2014)

18. Kiryakova, V.S.: Generalized Fractional Calculus and Applications. CRC Press, Boca Raton (1993)

19. Losada, J., Nieto, J.J.: Properties of a new fractional derivative without singular kernel. Prog. Fract. Differ. Appl. 1(2), 87-92 (2015)

20. Liu, W., Ngǒ, Q.A., Huy, V.N.: Several interesting integral inequalities. J. Math. Inequal. 3(2), 201-212 (2009)

21. Mubeen, S., Habib, S., Naeem, M.N.: The Minkowski inequality involving generalized k-fractional conformable integral. J. Inequal. Appl. 2019, 81 (2019). https://doi.org/10.1186/s13660-019-2040-8

22. Nisar, K.S., Qi, F., Rahman, G., Mubeen, S., Arshad, M.: Some inequalities involving the extended gamma function and the Kummer confluent hypergeometric k-function. J. Inequal. Appl. 2018, 135 (2018)

23. Nisar, K.S., Rahman, G., Choi, J., Mubeen, S., Arshad, M.: Certain Gronwall type inequalities associated with Riemann-Liouville $k$ - and Hadamard k-fractional derivatives and their applications. East Asian Math. J. 34(3), 249-263 (2018)

24. Set, E., Noor, M.A., Awan, M.U., Gözpinar, A.: Generalized Hermite-Hadamard type inequalities involving fractional integral operators. J. Inequal. Appl. 2017, 169 (2017)

25. Podlubny, I.: Fractional Differential Equations. Academic Press, London (1999)

26. Qi, F., Rahman, G., Hussain, S.M., Du, W.S., Nisar, K.S.: Some inequalities of Čebyšev type for conformable $k$-fractional integral operators. Symmetry 10, 614 (2018). https://doi.org/10.3390/sym10110614

27. Rahman, G., Nisar, K.S., Qi, F.: Some new inequalities of the Gruss type for conformable fractional integrals. AIMS Math. 3(4), 575-583 (2018)

28. Rahman, G., Nisar, K.S., Mubeen, S., Choi, J.: Certain inequalities involving the $(k, \rho)$-fractional integral operator. Far East J. Math. Sci.: FJMS 103(11), 1879-1888 (2018)

29. Rahman, G., Khan, A., Abdeljawad, T., Nisar, K.S.: The Minkowski inequalities via generalized proportional fractional integral operators. Adv. Differ. Equ. 2019, 287 (2019). https://doi.org/10.1186/s13662-019-2229-7

30. Sarikaya, M.Z., Budak, H.: Generalized Ostrowski type inequalities for local fractional integrals. Proc. Am. Math. Soc. 145(4), 1527-1538 (2017)

31. Sarikaya, M.Z., Dahmani, Z., Kiris, M.E., Ahmad, F.: (k, s)-Riemann-Liouville fractional integral and applications. Hacet. J. Math. Stat. 45(1), 77-89 (2016)

32. Set, E., Tomar, M., Sarikaya, M.Z.: On generalized Grüss type inequalities for k-fractional integrals. Appl. Math. Comput. 269, 29-34 (2015) 